\title{
Ovary in Inguinal Hernia
}

\section{${ }^{1}$ Sonal Kotdawala, ${ }^{2}$ Utkrant Patel, ${ }^{3}$ Balkrishna Tanna, ${ }^{4}$ Parul Kotdawala}

\begin{abstract}
Inguinal hernia is a very common condition. Ovary in inguinal hernia is a common condition for a pediatrician as the pain symptoms will necessitate an early visit to health facility in young childhood. Ovarian and fallopian tube inguinal hernias are commonly associated with defects in genital tract development.
\end{abstract}

Keywords: Hernia, Inguinal, Post-inflammatory implant.

How to cite this article: Kotdawala S, Patel U, Tanna B, Kotdawala P. Ovary in Inguinal Hernia. Int J Gynecol Endsc 2017;1(1):53-55.

\section{Source of support: Nil}

\section{Conflict of interest: None}

\section{INTRODUCTION}

Inguinal hernia is a very common condition. Ovary in inguinal hernia is a common condition for a pediatrician as the pain symptoms will necessitate an early visit to health facility in young childhood. But this is very uncommon for a gynecologist due to its rare presentation in the reproductive age. Most of the cases are already treated by the pediatricians/pediatric surgeons during childhood. Ovarian and fallopian tube inguinal hernias are commonly associated with defects in genital tract development. ${ }^{1}$ We report a case of a woman presenting with inguinal hernia with ovary and fallopian tube as its contents and unicornuate uterus.

\section{CASE REPORT}

A 19-year-old young unmarried girl presented at Kotdawala Women's Clinic complaining of swelling and pain of sudden onset in suprapubic region since 3 days. She gave a history that the swelling was small initially, and was increasing in size day by day. It was associated with dragging type of pain, which was continuous throughout and was gradually increasing in severity. Patient had normal bowel and bladder functions. Patient had normal regular menstrual cycles with menarche at the age of 12 years. She had her last menstrual period 10 days back. On examination, there was suprapubic swelling of about

\footnotetext{
${ }^{1,3}$ Consultant, ${ }^{2}$ Trainee, ${ }^{4}$ Director

${ }^{1-4}$ Kotdawala Women's Clinic, Ahmedabad, Gujarat, India
}

Corresponding Author: Parul Kotdawala, Kotdawala Women's Clinic, 53/1 BMM Society, Ahmedabad, Gujarat, India, e-mail: kotdawala@yahoo.com
$3 \times 4 \mathrm{~cm}$ on the left side (Fig. 1 ). It was hard in consistency with restricted mobility. The swelling was tender to touch and was irreducible. There was polydactyly instead of the thumb in left hand. Upon ultrasonography, it suggested hernia of the left ovary with diminished blood flow. There was normal uterus and right ovary. Abdominal sonography was normal. Patient was taken up for emergency laparoscopy with assistance of surgeon after obtaining consent (Figs 2A to C). Upon laparoscopy it was noticed that both inguinal canals were patent and open. Left inguinal hernia had left ectopic ovary and fimbrial end of the fallopian tube in its content. There was a unicornuate uterus. Right ovary and fallopian tube were normal and in their normal place. Peritoneum reflected off from the anterior abdominal wall; herniated ovary and fallopian tube retracted out of the inguinal canal. Inguinal canal defect was closed by placing polypropylene mesh by fixing it with the lateral wall with the endostapler. Retracted left ovary was suspended over the mesh on the anterior abdominal wall. Right inguinal ring (Fig. 3) was closed by taking approximating suture with Vicryl No. 2-0 as patient's relative did not give consent for prophylactic mesh repair. Upper abdomen was normal. Patient had uneventful postoperative period (Fig. 4). Patient was discharged from the hospital on postoperative day 2. Patient had normal examination and ultrasonography findings at the end of 2 months.

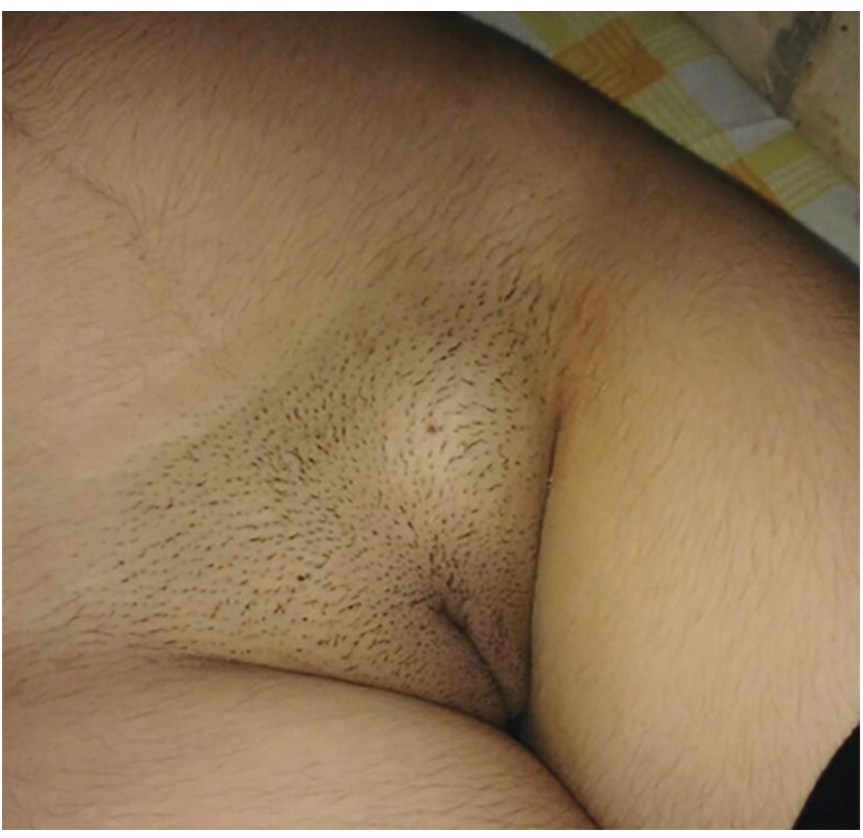

Fig. 1: Distinct swelling on It. inguinal region seen 


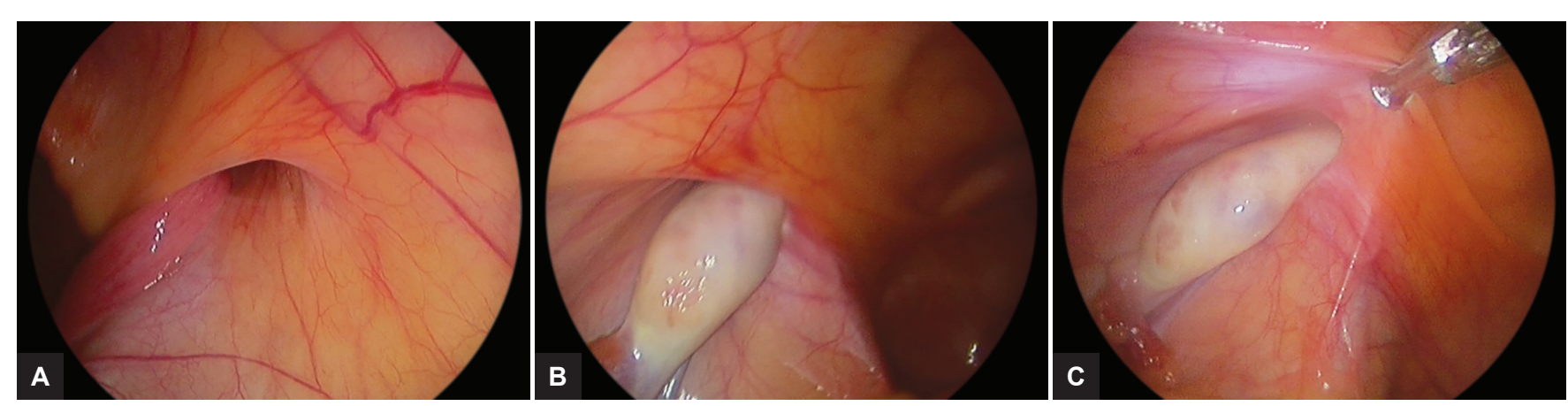

Figs $2 A$ to $C$ : (A) Open right inguinal ring, early hernia, (B) ovary in left internal inguinal ring; and (C) ovary in left internal inguinal ring

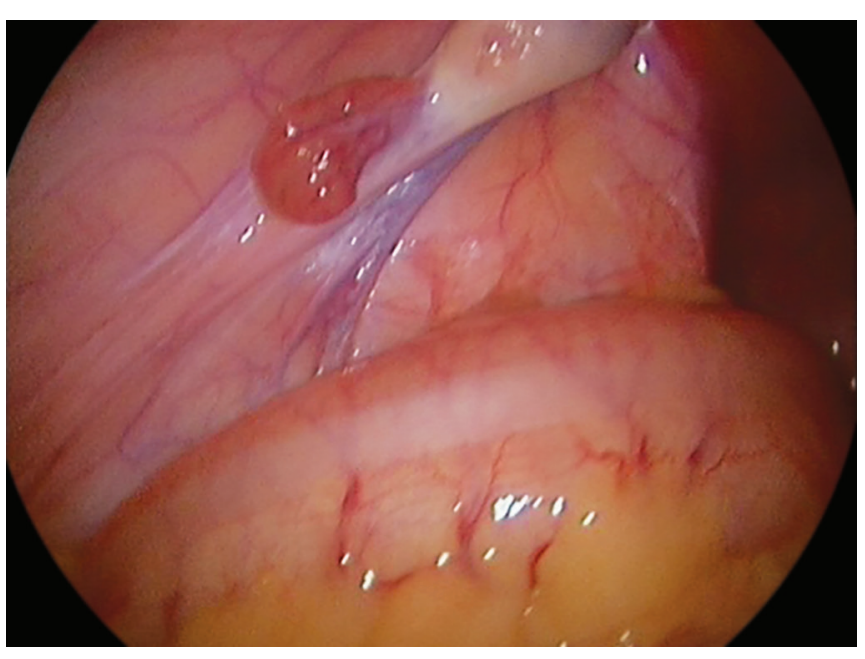

Fig. 3: Left ovary, tube \& infulndibulo-pelvic ligament pulled in the inguinal ring

\section{DISCUSSION}

Hernia of an ovary is rare, and $95 \%$ of ovarian hernias are inguinal. A variety of terms have been used to describe an undescended or ectopic ovary. In 1991, Lachman and Berman ${ }^{2}$ proposed a new terminology for classification of ectopic ovaries. They suggested that ectopic or undescended ovaries can be divided in three categories: (1) Postsurgical implant, (2) postinflammatory implant, and (3) true embryologic.

The association of unicornuate uterus and urinary tract anomalies including ectopic kidney, renal agenesis, double renal pelvis, and horseshoe kidneys is also well known. ${ }^{3}$

Normally the descent of the ovary through the canal of Nuck to the base of labium majus is prevented by the fixation of the gubernaculum of the ovary - the anlage of the ovarian and round ligaments - to the cornua of the uterus. The distal end of the gubernaculum is attached to the base of the labium majus. The canal of Nuck normally becomes obliterated by the end of the 8 th month of intrauterine life. If, however, the canal of Nuck remains open, shortening of the distal end of the gubernaculum or the change in the relative growth of the parts, together with failure of the attachment of the proximal end of the

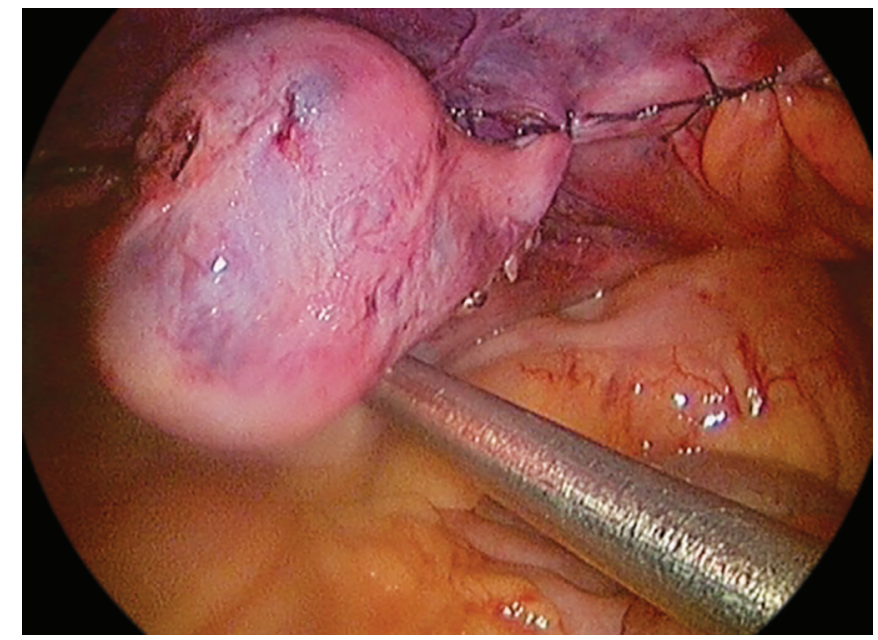

Fig. 4: Ovary in peritoneal cavity, end of surgery

gubernaculum in time to the deformed or underdeveloped uterus causes the ovary to be pulled into the canal of Nuck lying within the open inguinal canal. ${ }^{4}$

In 1941, Mayer and Templeton ${ }^{5}$ compiled and evaluated practically all the cases reported in the world literature and divided the 195 cases into those in which definite inguinal ectopia of the ovary appeared to be present (138) and those in which there was a questionable element (57). They reported that, of the 195 cases of tuboovarian hernias, $64 \%$ were in children under 2 years of age, and $71 \%$ in children under 5 years of age. They also observed that in true ectopia, the tube usually accompanies the ovary. Elliott et $\mathrm{al}^{6}$ reported a case of a woman with normal number of chromosomes and presence of uterus, fallopian tube, and ovary in an inguinal hernia associated with a unicornuate uterus.

Elliott et $\mathrm{al}^{6}$ reported a case of a woman with normal number of chromosomes $(46, \mathrm{XX})$ and presence of a uterus, fallopian tube, and ovary in an inguinal hernia associated with a unicornuate uterus.

Ombelet et $\mathrm{al}^{7}$ reported two cases of ectopic ovary associated with infertility and diagnosed during infertility workup. Both the cases had unicornuate uterus and ectopic ovary. 


\section{CONCLUSION}

Ectopic ovary is also associated with other urogenital maldevelopments. Early surgical intervention is preferred to prevent torsion. Inguinal canal closure is an important step to prevent occurrence of the same condition and future chance of hernias through inguinal canal. Patient should be counseled for the future fertility aspects and malignancy aspects to prevent adverse outcomes.

\section{REFERENCES}

1. Bradshaw KD, Carr BR. Ovarian and tubal inguinal hernia. Obstet Gynecol 1986 Sep;68(3 Suppl):50S-52S.
2. Lachman MF, Berman MM. The ectopic ovary. A case report and review of the literature. Arch Pathol Lab Med 1991 Mar; 115(3):233-235.

3. Fedele L, Bianchi S, Agnoli B, Tozzi L, Vignali M. Urinary tract anomalies associated with unicornuate uterus. J Urol 1996 Mar;155(3):847-848.

4. Donald DC. Ectopia and inguinal hernia of ovary. Am J Surg 1940 Jan;47(1):149-152.

5. Mayer V, Templeton FG. Inguinal ectopia of the ovary and fallopian tube: review of the literature and report of the case of an infant. Arch Surg 1941;43:397-408.

6. Elliott DC, Beam TE, Denapoli TS. Hernia uterus inguinale associated with unicornuate uterus. Arch Surg 1989 Jul;124(7):872-873.

7. Ombelet W, Verswijvel G, de Jonge E. Ectopic ovary and unicornuate uterus. N Engl J Med 2003 Feb;348(7):667-668. 
